\title{
Carne única, nueva carne. Cuerpo y democratización de tecnologías biomédicas de transformación corporal para personas trans en el actual escenario argentino
}

\section{| ${ }^{1}$ Maria Alejandra Dellacasa |}

Resumen: Este artículo pretende reflexionar acerca del proceso que va desde la producción de conocimiento biomédico en torno a identidades sexo-genéricas diversas, a las transformaciones en los modos de representar a las personas trans como sujetos de derecho, frente al Estado argentino. La intervención en la escena política de colectivos organizados imprimió una serie de particularidades al dispositivo médico-legal de la transexualidad en Argentina. Así, sujetos-pa(de)cientes nacidos en "cuerpos equivocados" pasaron a organizarse colectivamente y a emponderarse desde ese cuerpo como vehículo, para exigir y negociar otras posibilidades de ser y estar en el mundo. La reciente sanción de la Ley de Identidad de Género introdujo cambios en torno a la democratización del acceso a tecnologías biomédicas de transformación corporal, así como en las dinámicas de atención hospitalaria y sus implicancias en la producción de subjetividades mediadas biotecnológicamente. Finalmente, se introduce un debate en torno a nuevas formas de biosociabilidad y producción de conocimiento que configuran particulares relaciones entre ciencia, tecnología, usuarios, profesionales de la salud e instituciones públicas.

> Palabras clave: tecnologías biomédicas; producción de conocimiento; biosociabilidad; personas trans.
${ }^{1}$ Instituto de Geografía, Historia y Ciencias Sociales (IGHCSCONICET). Buenos Aires, Argentina (maledellacasa@yahoo.com.ar). ORCID: 0000-0002-0138-2501
Recebido em: 02/08/2017 Aprovado em: 10/07/2018 Revisado em: 20/08/2018 


\section{Introducción}

En este trabajo comenzaremos por desandar la delimitación de un campo específico de saber e intervención sobre los cuerpos, que aborda la transexualidad como una experiencia medicalizada. Trazamos un recorrido que va desde las iniciales categorizaciones biomédicas en torno a las identidades sexo-genéricas diversas, a las posibilidades de transformaciones corporales cada vez más complejas y abarcativas que ofrecen las tecnologías a las personas trans. ${ }^{1}$ Analizamos el proceso que parte de un sujeto pa(de)ciente nacido en un "cuerpo equivocado", al emponderamiento y posicionamiento desde ese cuerpo, como vehículo para exigir y negociar otras posibilidades de ser y estar en el mundo.

La perspectiva de análisis que abordamos se nutre de los aportes de la antropología de la salud y del campo de los estudios sociales de la ciencia y la tecnología (SSTS). Desde el año 2011 venimos desarrollando trabajo de campo con varios equipos de profesionales que brindan atención de la salud a personas trans en hospitales públicos de Argentina. ${ }^{2}$ A partir de realizar observación participante en espacios de atención, de la participación en reuniones previas al diseño de reglamentaciones y documentos y de diversas entrevistas en profundidad con profesionales de la salud, funcionarios públicos y militantes de colectivos trans, conformamos un corpus de registros de los que se desprende el análisis que aquí retomamos.

En este escrito de reflexión teórica profundizamos en torno a las particularidades que el dispositivo médico-legal de la transexualidad adquirió en Argentina, a partir de la intervención en la escena política de colectivos organizados en torno a identidades que se alejan de ciertos tintes patologizantes, sin desvincularse completamente de la medicalización. En tanto, es esta última instancia la que habilita el acceso a intervenciones corporales, hormonales y quirúrgicas en los servicios públicos de atención. En este sentido, recuperamos parte del análisis de Pecheny y de la Dehesa (2009) acerca de los cruces entre políticas públicas y sexualidades, con la finalidad de analizar el caso que nos ocupa.

La experiencia trans se presenta inicialmente atravesada por procesos de medicalización y judicialización, junto a una lógica de reconocimiento vinculada a la victimización y el sufrimiento individual. Luego, paulatinamente, ha devenido en una experiencia socializada y politizada a partir de espacios de participación y reconocimiento colectivos, que habilitan un rol activo de las personas en su 
negociación frente al Estado y a los profesionales de la salud. Dicho tránsito se dio paralelamente al proceso de discusión de la Ley de Identidad de Género en Argentina, cuya sanción y reglamentación imprimió profundas transformaciones en las dinámicas de atención en los hospitales, dando lugar a una serie de políticas públicas que se desprendieron de ello.

Finalmente, introducimos un debate en torno a nuevas formas de biosociabilidad (RABINOW, 1996) y producción conjunta de conocimiento que configuran particulares relaciones entre ciencia, tecnología, usuarios, profesionales de la salud e instituciones públicas.

\section{Categorizaciones biomédicas}

Habitualmente se llama "proceso moderno de medicalización" a las formas en que la jurisdicción médica inició una expansión extraordinaria y desde el siglo XVIII comenzó a abarcar muchos "problemas", que hasta ese momento no habían sido definidos como temas médicos. Foucault define la medicalización como "el hecho de que la existencia, la conducta, el comportamiento, el cuerpo humano, se incorporaran a partir del siglo XVIII en una red [...] cada vez más densa y más amplia, que cuanto más funciona, menos se escapa a la medicina” (1990, p. 122).

En los pasados dos siglos, una extensa gama de comportamientos han sido subsumidos bajo la rúbrica médica. La creciente obsesión por localizar los antecedentes genéticos de ciertos padecimientos y de diagnosticar y "tratar" algunos comportamientos considerados poco convencionales significan, en definitiva, que el saber basado en la medicina científica ha reforzado su control en la definición de los límites de la normalidad/ anormalidad, del apropiado funcionamiento del cuerpo y del comportamiento del ser humano ${ }^{3}$ (CONRAD, 1992).

La sexualidad no quedó exenta de esa conquista y a fines del siglo XIX y principios del siglo XX, vio la luz un campo de saber que tenía como finalidad específica el descubrimiento de las "leyes" y los parámetros de "normalidad" de la sexualidad humana: la sexología. El corpus de conocimientos que dicha especialidad produjo y produce, ha venido influenciando de manera definitiva el modo en que la sociedad concibe las prácticas y las sexualidades, en gran medida legitimado por tratarse de un conocimiento científico. Así surgieron nuevos expertos médicos, categorías nosológicas y diagnósticos que delimitaron ciertas prácticas, deseos y experiencias 
como "patológicos", habilitando la intervención biomédica ante las desviaciones, los tránsitos o las ambigüedades en el campo de la sexualidad. Este nuevo espacio de saber e intervención específica acerca de las conductas y los cuerpos, permitió comenzar a delimitar y constituir en un mismo movimiento aquellas "desviaciones" plausibles de "tratamiento", y por ende de "reencause", y aquellas que no lo eran. Las personas que manifestaban una identidad de género distinta de la categorización biomédica asignada en función de su genitalidad, fueron inicialmente diagnosticadas como transexuales y posteriormente identificadas como portadoras de un trastorno en la identidad de género (TIG), causado por una disforia. ${ }^{4}$

La aparición a mediados del siglo XX de la categoría género inauguró un nuevo paradigma. Si bien los trabajos de Money y Stoller en las décadas de los ’50 y ‘60 no fueron los primeros que habilitaron la posibilidad de distinguir entre el llamado "sexo biológico" y la "sexualidad psíquica", ya lo había hecho Freud en sus ensayos, fueron quienes popularizaron el concepto de género. John Money en 1952 introdujo el término "rol de género" y Robert Stoller (1968) habló por primera vez formalmente de "identidad de género". Partiendo de una perspectiva psicológica, articularon tres cuestiones básicas: la asignación de género (en el momento del nacimiento a partir de la apariencia externa de los genitales); la identidad de género (desarrollada entre los dos y tres años al momento de adquisición del lenguaje) y el rol de género (el actor social y sus roles resultan definidos por una particular interiorización de las normas y prescripciones que dictan la sociedad y la cultura sobre el comportamiento femenino o masculino).

Los desarrollos vinculados con la asepsia y las nuevas técnicas quirúrgicas, los descubrimientos acerca de las hormonas y el surgimiento del concepto de género, condicionaron en gran medida las concepciones modernas, los discursos y el tratamiento biomédico de lo que pasó a categorizarse como transexualismo o transexualidad (HAUSMAN, 1995). Las respuestas vinculadas a la intervención corporal que ofrecía la biomedicina para este fenómeno imprimieron decisivas transformaciones en el dispositivo de la transexualidad, promoviendo la "adecuación" corporal de lxs "pacientes", a partir de terapias de reemplazo hormonal y cirugías de "cambio de sexo". Entre 1960 y 1970, el dispositivo se consolida en base a la articulación entre discusiones teóricas y prácticas reguladoras de los cuerpos, con el nacimiento de asociaciones internacionales de médicos y pacientes, ${ }^{6}$ junto con la instauración de un protocolo de atención. ${ }^{7}$ La instancia definitiva que dio forma 
a un "síndrome" en el sentido moderno, fue la inclusión del transexualismo como patología psiquiátrica en el CIE (OMS, 1975) y en el DSM III (APA, 1980), aunando de este modo los criterios diagnósticos. ${ }^{8}$

Las instancias médicas de transformación corporal - tanto químicas como quirúrgicas - han tendido a reproducir el orden dimórfico junto con los ideales socialmente aceptados de lo que es "ser hombre" o "ser mujer"; a la vez que a imprimir una corporalidad signada por una serie preconceptos respecto de lo apropiado, lo adecuado y lo saludable (aunque no siempre lo funcional), en el terreno de la sexualidad. "Las ciencias son uno de los instrumentos más persuasivos que existen para convencer a los demás de qué son y qué deberían ser" (LATOUR, 1983, p. 143).

En este sentido, nos preguntamos cuáles son los parámetros inobjetables de lo femenino y lo masculino, producidos por los mecanismos de poder y las lógicas de consumo respecto de las imágenes corporales y su mediación tecnológica. En la mayoría de los casos, los profesionales de la salud abordan la experiencia transexual como un "trastorno mental" y las respuestas terapéuticas que implementan tienden a reorganizar subjetividades "apropiadas" retirando todos los indicadores de ambigüedades. En consonancia con el principio rector de que a cada persona le corresponde un sexo único, estable y concordante con un género - de dos posibles -, a lo largo de toda su vida; coincidimos con Roen (1998) en que las tecnologías biomédicas tendientes a "mejorar o corregir lo natural", siempre trabajan con reajustes dentro de normas consideradas "aceptables".

\section{La salvación por el cuerpo. Diagnosticando el género, despolitizando las sexualidades}

La posibilidad de construir una subjetividad mediada biotecnológicamente conduce a la paradoja del cuerpo como algo que se "es" y a la vez como algo que "se tiene temporalmente"; una intersección entre una "carne única" y la posibilidad de una "nueva carne" cuya transformación es parte de una relación atravesada inevitablemente por una serie de dispositivos de saber y poder.

En las narrativas de los profesionales acerca de la experiencia transexual, el cuerpo es relatado como algo "ajeno a uno mismo", algo que "nos es dado en gracia" y que se habita incómoda, angustiosa y parcialmente. Algunas personas trans narran la vivencia de sentirse "atrapadas en un cuerpo equivocado", que la naturaleza les ha 
proporcionado. Y en algunos casos se han apropiado del discurso patologizante y las categorías biomédicas, como vehículo para acceder al cambio de nombre y sexo en los registros oficiales y para solicitar intervenciones corporales, tanto hormonales como quirúrgicas frente al Estado. Tal como plantea Judith Butler (2009) "el diagnóstico" para algunos es una "bendición ambivalente, para otros una maldición ambigua”. Puede funcionar como un elemento que favorezca la autonomía y el deseo de transformación corporal de las personas transexuales, pero a la vez puede "asesinar el alma" (2009, p. 98).

El argumento "victimizante" patologiza e individualiza la experiencia trans, considerando que tiene lugar independientemente del contexto histórico y las transformaciones socioculturales que la atraviesan. Sin embargo, es el fundamento que prevalece en la mayoría de los países del mundo para hacer lugar a los pedidos de cambio registrales y al acceso a intervenciones corporales; siendo también el discurso que predominó en Argentina hasta la efectiva sanción de la Ley 26.743, conocida como Ley de Identidad de género (2012).

El sufrimiento, queen algunos casos se presenta aparejado a la experiencia transexual, es el argumento que se esgrime para justificar como "médicamente necesarias" las intervenciones corporales. Siguiendo el planteo de Fassin (2003), la biolegitimidad del cuerpo sufriente funciona como habilitante en la búsqueda y administración del "tratamiento". Las representaciones y los relatos que los profesionales de la salud construyen respecto de las personas trans, los presentan como sujetos sufrientes y cuánto más extremo es el sufrimiento y el rechazo del propio cuerpo, más credibilidad y compasión despiertan las narraciones de los pa(de)cientes.

Retomando el análisis de Fassin (2003) acerca de los usos políticos del cuerpo y el modo en que es puesto en escena y en narrativas, como la "única verdad" que tienen los actores para "hacerse valer" en determinado momento; propongo pensar la transexualidad como una experiencia medicalizada y judicializada, en relación a la política del sufrimiento. ${ }^{10}$ En sus trabajos acerca de los pedidos de residencia y asistencia sanitaria para refugiados en Francia, Fassin (1999; 2003) analiza el modo en que patología y sufrimiento se combinan para construir una argumentación patética que expone el cuerpo a la vista de los otros, junto al relato de sí. Constituyendo de este modo una biolegitimidad que se le otorga al cuerpo sufriente, una suerte de reconocimiento médico y social que se trata de "hacer valer". De manera análoga, 
muchas personas trans que han concurrido a los consultorios y juzgados construyeron una argumentación respecto de su "malestar", en torno al cuerpo, a la angustia y a la propia biografía. El sufrimiento, la falta de adaptabilidad, los problemas asociados a la discriminación y a la falta de inserción social, constituyen la base sobre la que los profesionales justifican como "medicamente necesarios" los tratamientos y las intervenciones corporales. "Conmover al auditor se vuelve un objetivo en sí mismo en la exposición de la demanda... mostrar que el cuerpo se resiste a la decadencia y que él es el centro de un proyecto de inserción" (FASSIN, 2003, p. 61).

En este sentido, la equidad, la autonomía y la justicia se soslayan en los relatos, la reivindicación de derechos queda vedada en una exposición individual del sufrimiento y de sí. Esa idea de sujetos vulnerables, "víctimas" de un "cuerpo equivocado", fue también el argumento y el recurso discursivo que funcionó para hacer lugar a las demandas en el campo legislativo. Desde los discursos jurídicos, las personas trans también han sido representadas como un "grupo social minoritario, vulnerable e históricamente discriminado que debe ser restituido en sus derechos" (CABRAL, 2008).

Mario Pecheny y Rafael de la Dehesa (2009) describen tres mecanismos que han favorecido el proceso de despolitización de la sexualidad y que en este trabajo hemos descripto más arriba, en relación a la experiencia transexual: la victimización (o victimismo), la medicalización y la judicialización. Tal como analizan los autores, estos mecanismos abonaron importantes avances en los derechos sexuales, en las reparaciones individuales y mejoraron la posición relativa de los grupos minoritarios; pero lo hicieron en base a la reducción de las demandas de derechos colectivos a pedidos individuales de tipo reparatorio y no a partir de considerar a éstas personas como plenos y legítimos sujetos de derechos.

Esta primera descripción de "sujetos sufrientes" que muchas personas trans esgrimen y que otros actores reproducen y refuerzan - profesionales de la salud, abogados, funcionarios públicos ${ }^{11}$ - comenzó a complejizarse en el avance de nuestro trabajo de campo y la propia dinámica que fue dando forma a las relaciones del colectivo LGBTI, ${ }^{12}$ las organizaciones y activistas trans con las instituciones públicas en Argentina. La imagen platónica del cuerpo como "la cárcel del alma" había comenzado a desdibujarse y a presentarse como un posible delegado que habilita la gestión, las prácticas y las intervenciones de otras posibilidades, en y desde el cuerpo. 


\section{La legislación argentina: despatologizando las identidades, politizando las sexualidades}

El concepto de ciudadanía biológica (ROSE; NOVAS, 2003) se presenta cargado de ideas de sufrimiento, supervivencia y vulnerabilidad; sin embargo cuando incorporamos la dimensión colectiva en el análisis se logra comenzar a trascenderlas. "Una minoría ha aprendido a negociar los términos de su inclusión social y económica usando el argumento de la vida" (PETRYNA, 2002:5, citado en ROSE, 2012). Así una serie de derechos y protecciones toman como fundamento la vida misma, que adquiere un nuevo "valor" potencialmente negociable. La ciudadanía biológica es tanto individual como colectiva (ROSE; NOVAS, 2003). Individual, en términos del propio cuerpo, de la experiencia somática en relación al self, y a como el yo se autopercibe y reconfigura a partir de ciertas vivencias vinculadas al malestar y al sufrimiento. Al mismo tiempo es colectiva, en tanto, recuperando el concepto de biosociabilidad de Paul Rabinow (1996), habilita a la conformación de colectividades en torno a una cierta identidad compartida. Tal como lo describen Rose y Novas (2003), el ciudadano biológico no es una construcción "desde arriba"; es producto de negociaciones y relaciones complejas en un entramado que combina co-ciudadanos, no-ciudadanos, profesionales de la salud, gobierno, instituciones públicas, legislación y tecnologías.

En Argentina la sanción de la Ley 26.743 (2012), conocida como Ley de Identidad de Género, constituye un caso paradigmático a nivel mundial en tanto garantiza a las personas que lo soliciten el acceso a intervenciones corporales de acuerdo al género autopercibido, mediante cirugías parciales o totales y/o terapias hormonales; sin requerir evaluación diagnóstica, ni autorización judicial. ${ }^{13} \mathrm{~A}$ través de dicha legislación, el estado argentino garantiza la identidad de género en dos sentidos: en términos jurídicos al autorizar el cambio de nombre y sexo en los registros oficiales, y en términos corporales en vinculación al campo biomédico, a partir de proveer acceso gratuito a una atención de la salud integral, incluidas intervenciones quirúrgicas y hormonales. ${ }^{14}$

La sanción de la Ley de Identidad de Género, trajo aparejadas otras cuestiones como la inclusión del tema de las diversidades sexuales en la agenda pública - lo que otorgó gran visibilidad a distintas organizaciones LGBTI - a la vez que se volvió imperioso implementar nuevas estrategias que permitieran garantizar el acceso a una atención de la salud no patologizante ${ }^{15}$ para las personas trans. Desde el Ministerio 
de Salud de la Nación, se trabajó activamente en distintos líneas: convocatoria a profesionales que ya venían trabajando en hospitales públicos e invitación a los que estuviesen interesados en conformar nuevos equipos, sensibilización de los trabajadores de la salud en temáticas de diversidad sexual y derechos, invitación a distintas organizaciones trans para participar del proceso de toma de decisiones, compra y suministro de hormonas en las farmacias de los hospitales y diseño, redacción y publicación de un protocolo de atención.

Luego de la presentación de cuatro proyectos y un largo proceso de debate, en mayo del año 2012 se logró la efectiva sanción de la Ley de Identidad de Género; a partir de la cual el estado argentino se presenta como garante del derecho a una identidad, cuya "verdad" ya no está anclada en el sexo o en el plano biológico, sino que se encuentra radicada en lo más profundo de la intimidad de las personas. El concepto de autopercepción que menciona el texto de la Ley se vincula con la idea de realización personal, en este sentido, el reconocimiento que se expresa de la diversidad, se refleja en la autonomía para la toma de decisiones y el acceso a intervenciones corporales.

El proceso de debate previo a la sanción de la Ley de Identidad de Género, así como todas las dinámicas vinculadas a la reglamentación y efectiva implementación en hospitales públicos, movilizó enormemente a las personas trans y a las distintas organizaciones de lucha por los derechos LGBTI. En este sentido, hacemos referencia a un proceso de colectivización de las experiencias y de politización de las identidades. ${ }^{16} \mathrm{Si}$ bien inicialmente el lenguaje biomédico y la emergencia de categorías diagnósticas, promovieron subjetividades patológicas mediadas (bio) tecnológicamente para las personas trans. No es menos cierto, que la paulatina y creciente conformación de espacios colectivos de intercambio, organización y lucha, les han aportado - y lo siguen haciendo - un marco de pertenencia grupal; brindando innumerables y valiosas herramientas para la participación y el emponderamiento en la lucha por los derechos, modelando y politizando dicha subjetividad.

\section{Biosociabilidad trans. Sujetos políticos, sujetos sexuales, hacia un proceso de democratización}

Si la sociobiología es cultura constituida sobre la base de una metáfora de la naturaleza, entonces en la biosociabilidad la naturaleza resulta moldeada por la cultura entendida como práctica. El conocimiento técnico de la naturaleza permitirá remodelarla y final- 
mente se convertirá en artificial, de la misma manera que la cultura se transforma en natural. Si este proyecto se concretara, sería la base que permitiría superar la división entre naturaleza y cultura (RABINOW, 1996, p. 99).

Una experiencia, un malestar, o en este caso asumir una identidad sexual "disidente", devienen en un rol social que tiene incidencia en la interacción con otros, es decir, en cómo las personas se presentan y describen a sí mismas y cómo son descriptas, percibidas y representadas por otros. En este sentido, los "ciudadanos biológicos” encarnan subjetividades que establecen un cierto tipo de relación con sus cuerpos y con las tecnologías a partir de la incorporación y/o resistencia de discursos estigmatizantes/patologizantes. Dichos sujetos utilizan lenguaje biomédico para describir aspectos de sus experiencias, o de su identidad; a la vez que su organismo, su vida y su cotidianeidad se presentan atravesados por intervenciones (farmacológicas, quirúrgicas, estéticas, etc.) que los posicionan particularmente, respecto de cómo podrían/deberían actuar y del tipo de vida al que podrían aspirar.

Rabinow (1996) afirma que frente a esta nueva forma de bio-identidad, las tradicionales clasificaciones bioculturales en torno al género, la raza y la edad irán perdiendo poder. Así, nuevas clasificaciones biomédicas funcionan como organizadoras de nuevas identidades y grupalidades que involucran de algún modo conocimiento médico y científico; dando lugar a espacios tanto de negociación individual y gestión privada de opciones, como a espacios de disputa pública sobre experiencias corporales mínimas: celíacos, obesos mórbidos, portadores de patologías minoritarias, parejas infértiles, víctimas de ciertas catástrofes o accidentes, etc.

Alrededor de los procesos de atención se expresa también la agencialidad del sujeto, su capacidad de integrar - en el despliegue de estrategias - diferentes expectativas y prioridades, o de gestionar los conflictos entre diferentes economías morales (THOMPSON, 2000). Los procesos de atención y las opciones terapéuticas se insertan de forma compleja en las estrategias sociales del sujeto, en su vida, y se capilarizan en el modo en que se constituye su self, en el seno de sus relaciones sociales y en la relación con los profesionales. Como analizamos para el caso de las personas trans, los grupos de pertenencia (colectivos, organizaciones militantes, etc.) constituyen elementos importantes en la configuración de las subjetividades y en la agencia de los sujetos acerca de sus derechos y el acceso a la atención de la salud. Los vínculos y los intercambios con otrxs permiten compartir experiencias, 
hacer lobby, brindar información a compañerxs y familiares, y diseñar estrategias que permitan adaptarse mejor al contexto y a las necesidades de cada persona. Varios blogs y páginas web ofrecen autobiografías de personas que han decidido atravesar diferentes procesos de transformación corporal y narran parte de las estrategias que han desarrollado para acceder a la atención, o para conseguir ciertos fármacos y las diferentes experiencias que se desprenden de su consumo.

Varios grupos de sujetos congregados en torno a un malestar o una condición, se autoidentifican con nombres que hacen referencia a su experiencia y desarrollan un rol activo para ampliar y mejorar su conocimiento -particularmente el médicocientífico - respecto de su vivencia. El conocer explicaciones biológicas de una experiencia o nuevos desarrollos tecnológicos, puede servirles para mejorar las estrategias de resilencia y ampliar su propia comprensión de lo que les sucede. Las personas bien informadas están en mejores condiciones para negociar con los profesionales respecto de las opciones terapéuticas y las posibilidades de intervención. En este sentido Rose y Novas (2003) refieren a un creciente compromiso de algunas personas, en un proceso de (auto)formación biomédica; proceso que se encuentra ampliamente potenciado por las posibilidades que habilitan las nuevas tecnologías como fuentes de recursos e información.

Por otro lado, en lo que respecta al impacto de la democratización de los procesos de producción de conocimiento en la subjetivación de lxs "pacientes", las personas mejoran sus experiencias y clarifican una caracterización de lo que les sucede. Adquieren más información respecto de la posible evolución y aprenden a vincular la experiencia individual y las relaciones sociales, con los datos biomédicos y las tecnologías disponibles en una relación dinámica. Dicho discurso combina lo biológico y lo social para producir lo que Paul Rabinow (1996) llama biosociabilidad. La identidad individual y la del colectivo, de la que el sujeto pasa a ser un miembro, se configuran simultánea y mutuamente. Estas comunidades biosociales ponen en práctica una nueva ética informada (ROSE, 2012) que implica una serie de técnicas para manejar la vida diaria en relación a una condición o experiencia, y en relación con un conocimiento experto - en este caso el biomédico -, en tanto actúa como la puerta de acceso a diferentes opciones fundamentalmente ancladas en intervenciones corporales. 


\section{Nuevas formas de interacción entre tecnología y sociedad que se proyectan en el campo de la atención de la salud y de tecnologías aplicadas al cuerpo}

En los procesos de atención entran en juego signos, síntomas, índices, malestar y otras experiencias que se refieren a un cuerpo, lo atraviesan, a la vez que lo exceden en un plano diacrónico, que es el transcurso de la vida misma de lxs sujetxs. En este sentido, es difícil pensar la atención como un ejercicio aislado de gestión, o un acto individual de elección, por parte del paciente/usuario y de implementación/ ejecución por parte de los médicos. Para reemplazar el término "gestionar", Mol (2008) propone el término co-doctoring (shared doctoring) que condensa los esfuerzos por democratizar el conocimiento experto, compartir experiencias y sostener una práctica de intervenciones compartidas en lo que respecta a la atención de la salud (2008:56). En este sentido, el concepto de co-doctoring pretende dar cuenta de aquellos procesos atravesados por una actitud colaborativa entre las personas que concurren a los consultorios y los profesionales, con una postura abierta y receptiva de éstos últimos hacia las propuestas, saberes y experiencias de otras y otros. En un "vuelco simétrico", los especialistas y profesionales que participan de estas relaciones se ven empujados a considerar "el punto de vista del paciente". ${ }^{17}$ Lo que repercute también, en la conformación de la subjetividad y la carrera de los propios trabajadores de la salud (DELLACASA, 2013).

En el trabajo de campo, los profesionales nos han referido que muchas personas trans se presentan en los espacios de atención con una postura activa, predisposición hacia la cooperación, e intenciones de participación en las consultas, toma de decisiones y administración de las opciones terapéuticas.

En su trabajo Reseach “in the wild', shaping new social identities, Callon y Rabeharisoa (2003) refieren a nuevas formas de interacción entre tecnología y sociedad, en las que a partir de dinámicas de investigación biomédica cooperativas, los no científicos trabajan con los científicos para producir y promover el conocimiento.

Tal como sostiene Sheila Jasanoff, la co-producción de conocimiento implica que tanto el orden natural como el orden social son producidos conjuntamente y que ninguno de los órdenes prevalece por encima del otro (JASANOFF, 2004). Los saberes de legos y expertos en el campo de la salud los coloca en dos lugares muy distantes: socialmente se ha categorizado a los "expertos" como los especialistas 
(investigadores, profesionales de la salud) de los distintos campos disciplinares que generan y producen saber científico, y como "legos" en principio, se ha ubicado a los pacientes, quienes vivencian la experiencia. Desde una concepción constructivista del conocimiento como producto social, asumimos con dicha propuesta, el supuesto de que esta coproducción de conocimiento existe realmente, como un fenómeno que es tanto proceso como resultado de la interacción entre comunidad científica y sociedad.

Pareciera, tal como lo relata Mol (2008), que se abren dos caminos posibles para las personas trans como usuarias del sistema de salud, o se apoyan en una postura de autoexclusión y se vuelen en contra de una "ciencia" que se les presenta como distante, normalizadora y reductivista, que les impone etiquetas diagnósticas; o deciden desarrollar su propia capacidad de agencia, comenzando a participar y negociar, individual y colectivamente, en los procesos de categorización, desarrollo biotecnológico y producción de conocimiento con los expertos.

El protocolo de Atención de la salud integral para personas trans. Guía para equipos desalud, publicado recientemente por el Ministerio de Salud de Argentina, constituye una experiencia muy rica que se vincula directamente con esa idea de co-producción. Dicha guía que se desprende de la regulación de la Ley 26.743 (2015), fue producto de un largo y provechoso trabajo de colaboración entre los integrantes de tres equipos de profesionales de la salud que atienden a personas trans en hospitales públicos y de distintxs activistas y organizaciones de travestis, transexuales y transgénero de Argentina ${ }^{18}$ (además de los funcionarios públicos del ministerio que oficiaron como vínculo y articularon las reuniones y los espacios de encuentro).

Siguiendo con el paradigma despatologizante y de derechos humanos inaugurado por la Ley 26.743, la Guía recupera la autodeterminación de la posición de género y la autonomía de las personas para decidir sobre su propio cuerpo, como parte de los derechos sociales básicos, ya expresado en los Principios de Yogyakarta (2007). El documento hace hincapié en una serie de principios que apuntan claramente a sensibilizar a los profesionales de la salud - y a la sociedad toda-, además de mejorar las dinámicas de interacción en los espacios de atención. Se destaca en la redacción del texto el reconocimiento de la multiplicidad de las identidades, expresiones y trayectorias de género, a la vez que se subraya la singularidad en los modos en que cada persona vivencia y transita las identidades de género. Por otro lado, se plantea un abordaje de la salud desde una mirada integral y una atención de calidad, no discriminatoria y libre de cualquier forma de violencia. 
Identificamos una paulatina transformación de la lógica de producción de categorías diagnósticas y de conocimiento, así como del funcionamiento de éstas como condicionantes para el reconocimiento del derecho a la atención de la salud y el acceso a tecnologías de intervención corporal, mediadas por el saber biomédico. En este sentido, las definiciones diagnósticas, las intervenciones tecnológicas y las prácticas de atención comienzan a incluir no sólo a los profesionales de la salud (médicos clínicos, urólogos, cirujanos, psiquiatras, entre las principales especialidades médicas implicadas); sino también a las personas que demandan atención y que son portadoras de una serie de capacidades, experiencias y saberes acerca de lo que ellas mismas experimentan y desean, reconociéndole de este modo "su parte" en la responsabilidad respecto de la (de)construcción de categorías diagnósticas, de la toma de decisiones y las elecciones respecto de las intervenciones corporales.

\section{A modo de cierre}

Las subjetividades mediadas biotecnológicamente son parte de una construcción, un proceso más bien dinámico, dialéctico e incluso conflictivo, que monolítico. La idea de subjetividad remite a la compleja combinación de naturaleza y cultura, anunciando a la vez el fin de esos conceptos concebidos separadamente; dinamitando la visión estructuralista de que existe una ley, estructura-estructurante, para pensar la psiquis y la cultura humanas (DERRIDA, 1989). En este sentido, ciertas propuestas contemporáneas que abordan las subjetividades quedan dislocadas de los binomios modernos fundantes: naturaleza/cultura, sujeto/objeto, individuo/ sociedad, natural/artificial.

Tal como analizamos en este trabajo, las demandas colectivas que son producto de luchas y procesos más amplios de organización y movilización política imprimen características particulares a las condiciones y actores sociales que las posibilitan. La emergencia de sujetos que proponen una definición propia de sus experiencias y plantean soluciones en la medida que las consideran adecuadas, evidencia una matriz política que atraviesa y excede la experiencia de sufrimiento y el cuerpo individual. Una subjetividad política, colectiva, agenciada en la lucha y una capacidad de producción de identidades cuerpos y conocimientos "en situación” (HARAWAY, 1991). En este sentido, la elección y la autonomía se proyectan no desde una posición de ciudadanía que obvia la corporalidad en el modelo de sujeto autónomo y racional, 
sino precisamente en un ejercicio de reivindicación desde el cuerpo. Es éste el que se convierte en un campo de batalla y en el epicentro de las intervenciones.

\section{Referencias}

BUTLER, J. Desdiagnosticando o gênero. Physis Revista de Saúde Coletiva. Rio de Janeiro, v. 19, n. 1, p. 95-126, 2009.

CABRAL, M. Comparecer: un comentario, 2008. Disponível em: <http://www.clam.org.br/ uploads/conteudo/artigo_comparecer.pdf> Acesso em: 07/12/2016.

CALLON, M.; RABEHARISOA, V. Research "in the wild" and the shaping of new social identities. Technology in society, v. 25, p. 193-204, 2003.

CONRAD, P. Medicalization and social control. Annual Review of Sociology, n.18, p. 209232, 1992.

DELLACASA, M. A. Disputas en torno a la despatologización: sujetos-colectivos trans y expertos hacia un proceso de co-producción de conocimiento. En: REUNIÓN DE ANTROPOLOGÍA DEL MERCOSUR, XI., Montevideo, Uruguay, 2015.

. Un abordaje antropológico de los procesos diagnósticos protocolos y rutinas de intervención terapéutica en personas transexuales. Tesis (Maestría) - Facultad de Filosofía y Letras, Universidad de Buenos Aires. Inédito, 2013.

DERRIDA, J. La escritura y la diferencia. Barcelona: Anthropos, 1989.

FASSIN, D. Gobernar por los cuerpos, políticas de reconocimiento hacia los pobres y los inmigrantes en Francia. Cuadernos de Antropología Social, Buenos Aires, n. 17, p. 49-78, 2003.

. L'indicible et l'impensé. La "question immigrée" dans les politiques du sida. Sciences Sociales et Santé, v. 17, n. 4, p. 5-36, 1999.

FOUCAULT, M. El nacimiento de la clínica. Buenos Aires: Siglo XXI, 1990.

HARAWAY, D. Ciencia, cyborgs y mujeres: la reinvención de la naturaleza. Madrid: Cátedra, 1991.

HAUSMAN, B. Changing sex: transexualism, techonology and the idea of gender in the 20th century. Durham: Duke University Press, 1995.

JASANOFF, S. States of knowledge. The co-production of science and social order. Londres: Routledge, 2004.

LATOUR, B. Nunca hemos sido modernos. Madrid: Debate, 1993.

MOL, A. The logic of care: health and the problem of patient choice. London: Routledge, 2008. 
PECHENY, M.; DE LA DEHESA, R. "Sexualidades y políticas en América Latina: un esbozo para la discusión”. Trabalho apresentado no Diálogo Latinoamericano sobre Sexualidade e Geopolitica, Rio de Janeiro, ago. 2009. Disponível em: <http://www.sxpolitics.org/pt/wpcontent/uploads/2009/10/sexualidades-y-politicas-en-america-latina-rafael-de-la dehesa-ymario-pecheny.pdf>. Acesso em: $07 \mathrm{dez} .2016$.

RABINOW, P. Essays on the Anthropology of Reason. New Jersey: Princeton University Press, 1996. ROEN, K. But we have to do something: Surgical "correction" of atypical genitalia. Body \& Society, v. 14, n. 1, p. 47-66, 2008.

ROSE, N. Políticas de la vida. Biomedicina, poder y subjetividad en el siglo XXI. La Plata: Editorial Universitaria, 2012.

ROSE, N.; NOVAS, C. Biological Citizenship. In: ONG, A.; COLLIER, S. (Eds.). Blackwell Companion to Global Anthropology. Oxford: Blackwell, 2003.

THOMPSON, E. Costumbres en común. Estudios en la cultura popular tradicional. Barcelona: Crítica, 2000.

\section{Documentos}

Atención de la salud integral para personas trans. Guia para equipos de salud. Programa Nacional de salud sexual y procreación responsable. Ministerio de Salud. Presidencia de la Nación Argentina, 2015.

Ley de Identidad de Género. Ley n ${ }^{\circ} 26.743-n^{\circ} 32.404$, Boletín Oficial de la República Argentina, 24 de Mayo de 2012.

Principios de Yogyakarta. Principios sobre la aplicación de la legislación internacional de derechos humanos en relación con la orientación sexual y la identidad de género, 2007.

Reglamentación del artículo 11 de la Ley 26.743 sobre el Derecho a la Identidad de Género, Decreto Nacional n 903/2015, Boletín Oficial de la República Argentina, 29 de Mayo de 2015.

\section{Notas}

${ }^{1}$ Si bien el término transgénero es el más utilizado en el ámbito académico, en este trabajo utilizaremos el término trans que incluye a todas las personas cuyo sentido y autopercepción de la identidad de género difiere de la categoría asignada en el proceso biomédico de sexuación al nacer: travestis, transexuales y transgénero. A su vez, cuando en este trabajo hacemos referencia a las personas trans nos referimos particularmente a aquellas que concurren a los espacios de atención e interactúan con los profesionales de la salud. Con ello no pretendemos abordar la subjetividad y los deseos de la totalidad del colectivo trans, por el contrario, reafirmamos y celebramos su diversidad. 
${ }^{2}$ Este trabajo forma parte de la investigación vinculada a mi tesis de doctorado en Antropología Social de la Universidad de Buenos Aires, que se encuentra actualmente en proceso de finalización. Los estudios de doctorado fueron realizados mediante una beca doctoral del Consejo Nacional de Investigaciones Científicas y Técnicas de Argentina (CONICET).

${ }^{3}$ Todo ello habilitado por la reordenación epistemológica del siglo XIX, el paso de la medicina clásica a la medicina moderna. Ahora el objeto de saber es una cosa concreta, pero a la vez, independiente de su visibilidad, es preciso penetrar en el interior de los cuerpos para conocerlos.

${ }^{4}$ En este cambio de categorías, que identificamos en la historización del dispositivo de la transexualidad, puede apreciarse la influencia de la psiquiatría en la conformación de una estrategia y pasos formales para el diagnóstico, por otro lado puede analizarse la impronta del concepto de género. En tanto, se pasa de un trastorno de la sexualidad, a categorizarlo como un trastorno de personalidad/identidad.

${ }^{5}$ En 1962 la nueva edición del Oxford English Dictionary, afirma que "sexo" y "género" ya no son más términos equivalentes.

${ }^{6}$ En 1964 surgen la Erikson Educational Foundation (Louisiana) y la Harry Benjamin Foundation, ambas organizaciones filantrópicas tienen como objetivos la difusión, investigación médica y formación educativa acerca de la transexualidad como "problema médico". A partir de ellas se organiza la Unidad Nacional de Ayuda a los Transexuales, el primer Congreso médico acerca de la temática (Londres, 1969) y se publica el primer Boletin Trans.

${ }^{7}$ Standards of Care for Gender Dysphoria - 1ra. Versión - Asociación Internacional de Disforia de Género "Harry Benjamin", 1969.

${ }^{8}$ Si bien el diagnóstico se plantea configurado por la psiquiatría, las opciones terapéuticas y las intervenciones se anclaban en las transformaciones corporales - tanto quirúrgicas como hormonales - que practicaron inicialmente los equipos de Christian Hamburger en Dinamarca (1953) y Harry Benjamin en Estados Unidos (1966).

'Aquí, al igual que en el título, hacemos alusión a las expresiones "carne única" y "nueva carne" que corresponden a Thomas Laqueur, Historia de la sexualidad de los griegos a Freud (1990) y al cineasta David Cronenberg, Videodrome (1982), respectivamente.

${ }^{10}$ Didier Fassin $(1999,2003)$ plantea un movimiento desde la "política de la piedad" analizada por Hannah Arendt (1963) a la "política del sufrimiento" signada por una introducción del pathos en lo político, una patetización del mundo.

${ }^{11}$ La presentación por medio de abogados de recursos de amparo ante la justicia, era el modo en las personas previamente diagnosticadas como transexuales o intersexuales podían acceder a cambios de nombre y sexo en los registros oficiales y/o a intervenciones corporales - hormonales o quirúrgicas -, cuando comenzaron a practicarse en hospitales públicos en 2001. Para ampliar ver DELLACASA, M. Violencia de Estado: el reconocimiento de las personas transexuales como ¿sujetos "patológicos" de derechos? Revista Maguaré, v. 28, n. 1, Nacional de Colombia, p. 113-137, 2014.

${ }^{12}$ Las siglas LGBTTTI refieren a lesbianas, gay, bisexuales, travestis, transexuales, transgénero e intersexuales.

${ }^{13}$ A pesar del carácter progresista y despatologizante de la ley, no se plantea en el fondo una propuesta superadora en torno a los binarismos de género.

${ }^{14}$ En Argentina, un antecedente importante en vinculación con una mirada despatologizante es la sanción de la Ley 26.657 de Salud Mental (2010) que en su artículo $3^{\circ}$ inciso d) prohíbe hacer diagnósticos en el campo de la salud mental sobre la base de la identidad de género y de la identidad sexual. 
${ }^{15} \mathrm{El}$ antecedente más importante en este sentido es el movimiento Stop Trans Pathologization-STP, que viene organizando diferentes actividades con el objetivo de impulsar acciones de despatologización trans. Inicialmente, las primeras campañas surgieron de activistas españoles y a partir del año 2009 la plataforma virtual cobró relevancia internacional incorporando progresivamente más de 370 redes y organizaciones de los cinco continentes. Particularmente en la Campaña SPT-2012, en coincidencia con el proceso de discusión y producción de la $5^{\circ}$ versión del DSM, se han planteado marchas simultáneas en distintas partes del mundo con la consigna de eliminar definitivamente cualquier categorización biomédica para la transexualidad; proponiendo a la vez, un nuevo modelo relacional entre profesionales, tecnologías y personas trans. ${ }^{16}$ A partir de un juego de palabras hacemos alusión indirectamente a los aportes que los desarrollos en el campo de la antropología, podrían hacer a esta perspectiva de análisis. Latour (1993) Nunca hemos sido modernos y "Desde el punto de vista del nativo": sobre la naturaleza del conocimiento antropológico. En: GEERTZ, C. Conocimiento local. Ensayos sobre la interpretación de las culturas. Paidos, Barcelona, 1994.

${ }^{17}$ Entre estas nuevas dinámicas de investigación co-gestionadas, uno de los principales antecedentes, es la Asociación Francesa contra la Myopathies.

${ }^{18}$ Entre lxs convocadxs y las colaboraciones en la redacción de Atención de la salud integral para personas trans. Guia para equipos de salud, figuran varixs activistxs y las organizaciones: Futuro Transgénico, Asociación Travestis, Transexuales y Transgénero de Argentina (ATTTA) y la Red Intersexual, Transgénero y Transexual de Argentina (RITTA). 


\section{Abstract}

Unique meat, new meat. Body and

democratization of biomedical technologies of corporal transformation for trans people in the current Argentine scenario

This paper looks to reflect on the process ranging from the production of biomedical knowledge around diverse sex-generic identities, to the transformations in the ways of representing trans people, as subjects of law in the Argentine State. The organizations have broken into the political arena, imprinting some characteristics to the medical and legal transsexuality device in Argentine. Thus, subjects-patients/sufferers that have born in "wrong bodies" became collectively organized and empowered from this body as a vehicle to demand and negotiate other possibilities of being in the world. The recent approval of the Gender Indentity Law introduced changes in the democratization of access to biomedical technologies of body transformation, in the hospital care dynamics and its implications on the production of subjectivities biotechnologically mediated. Finally, we introduce the discussion about new ways of biosociabilty and knowledge production, which configure particular relationships between science, technology, users, health professionals and public institutions.

> Keywords: biomedical technologies; knowledge production; biosociability; trans people. 


\section{Resumo}

Carne única, carne nova. Corpo e

democratização das tecnologias biomédicas de transformaçâo corporal para pessoas trans no atual cenário argentino

Este artigo tem como objetivo refletir sobre o processo que vai desde a produção de conhecimento biomédico sobre diversas identidades sexuais genéricas, até mudanças nos modos de representar as pessoas transexuais como sujeitos de direito frente ao governo argentino. A intervenção dos coletivos organizados no cenário político imprimiu uma série de particularidades ao dispositivo médico-legal da transexualidade na Argentina. Assim, sujeitos-pacientes / que padecem, nascidos em "corpos errados", passaram a se organizar coletivamente e empoderar-se a partir daquele corpo como um veículo para exigir e negociar outras possibilidades de ser e estar no mundo. A recente promulgação da Lei de Identidade de Gênero introduziu mudanças na democratização do acesso a tecnologias biomédicas de transformação corporal e dinâmicas de cuidados hospitalares e suas implicações na produção de subjetividades mediadas biotecnologicamente. Por fim, apresenta-se um debate em torno de novas formas de biossociabilidade e produção de conhecimento que formam relações particulares entre ciência, tecnologia, usuários, profissionais de saúde e instituiçôes públicas.

> Palavras-chave: tecnologias biomédicas; produção de conhecimento; biossociabilidadae; pessoas trans. 